\title{
The Pain Prevalence, Characteristics and Related Factors of Non-Small Cell Lung Cancer (NSCLC) Referral to Palliative Care Service
}

\section{Xiaoli Gu}

Fudan University Shanghai Cancer Center

\section{Huifeng Gao}

Fudan University Shanghai Cancer Center

\section{Menglei Chen}

Fudan University Shanghai Cancer Center

Minghui Liu

Fudan University Shanghai Cancer Center

\section{Zhe Zhang}

Fudan University Shanghai Cancer Center

\section{Wenwu Cheng ( $D$ wenwucheng@yahoo.com )}

Fudan University Shanghai Cancer Center https://orcid.org/0000-0001-8923-1857

\section{Research article}

Keywords: advanced non-small-cell lung, cancer pain, epidermal growth factor receptor, epidermal growth factor receptor-Tyrosine kinase inhibitors, palliative care

Posted Date: July 6th, 2020

DOl: https://doi.org/10.21203/rs.3.rs-40071/v1

License: (c) (1) This work is licensed under a Creative Commons Attribution 4.0 International License. Read Full License 


\section{Abstract}

Background冈This study is to analyze the occurrence and clinical characteristics of cancer pain in advanced non-small cell lung cancer (NSCLC) patients who referral to palliative care treatment, as well as correlation between pain performance and clinical characteristics, laboratory indicators, and previous treatment.

Methods XAdvanced NSCLC patients referred to the Palliative Care Unit (PCU) from January of 2016 to December of 2018 were included in our study. The Brief Pain Inventory (BPI) was used to evaluate cancer pain (CP).

Results: Of 54 (56.7\%) patients reported with pain, there were 23 patients $(42.6 \%)$ who suffered from severe pain (score $>=7$ ) at the admission. There was a significant difference in the frequency of pain between patients with and without bone metastasis $(p=0.006)$, between patients with more than two sites and non-more than two sites metastasis $(p=0.032)$. Patients received epidermal growth factor receptor-tyrosine kinase inhibitors (EGFR-TKI) had lower pain prevalence $(p=0.033)$. Patients with bone metastases $(p=0.005)$, with more than two metastases $(p=0.022)$ and those with higer $C$-reactive protein (CRP) index $(p=0.012)$ had higher mean scores of pain, with significant differences.

\section{Conclusions:}

Our study showed the general characteristics, pain performance and related factors of advanced NSCLC patients who referral to palliative care service. This study confirmed that these patents' clinical manifestations of pain were correlated with bone metastasis, metastasis with more than two sites, elevated CRP, and the EGFR-TKI usage. Whether there is a certain potential connection between these factors deserves further study.

\section{Background:}

Lung cancer is one of the most serious malignant tumors, and its incidence rate and mortality rate has been ranked [1]. For advanced lung cancer patients, pain is one of the most troubling symptoms of their disease. The incidence of cancer pain(CP) was reported to be $64 \%$ in cancer patients[2]. CP continued to affect $33 \%$ of cancer patients even after anti-pain treatment. Even after receiving pain relief treatment, $30 \%$ of patients still suffer from cancer pain [3, 4]. During the whole course of cancer disease, patients are more or less disturbed by pain.

The primary site of tumor, local invasion, distant metastasis sites and previous treatments should be considered as the causes of lung cancer patients' pain. CP may also be due to peripheral growth of tumor (e.g. pleural or chest wall involvement) or nerve involvement (arm or shoulder pain, typical pancoast syndrome). Some visceral pain was unrelated with the invasion of local structures [5]. Moreover, CP may be unrelated to cancer itself, but to the systemic inflammatory response status of patients, especially for the elderly patients. 
For advanced lung cancer patients, especially non-small cell lung cancer (NSCLC) patients, gene detection is very important. For patients with gene mutation, targeted therapy is the first-line treatment recommended by the current guidelines [6]. Since epidermal growth factor receptor-tyrosine kinase inhibitors (EGFR-TKI) has been first-line recommended in NSCLC patients with epidermal growth factor receptor (EGFR) mutation and has been widely used, patients' baseline situation had been affected who referal to palliative care. Clinical literatures indicated that EGFR-TKI may have analgesic properties [7]. EGFR-TKI were testified to attenuate neuropathic pain in humans in previous research [8]. The effect of EGFR mutation and the EGFR -TKI on cancer pain in NSCLC are worth study.

A large number of literatures reported the current situation of CP in advanced cancer patients, but few studies have focused CP characteristics of specific single disease patients, including common cancers such as NSCLC [9]. The objective of this study is to determine the ouucrance and clinical characteristics of CP in NSCLC patients who referral to palliative care treatment, as well as laboratory indicators, previous treatments and other related factors.

\section{Methods}

\section{Samples and procedures}

Advanced NSCLC patients referred to the PCU from January of 2016 to December of 2018 were included in our study. Patients with incomplete general information were excluded from our study. Patients who were survived until the data collection were analyzed as censor. The data of 95 patients with definite pathology were finally included in the analysis. We followed the ethical guidelines of the FUSCC review board. As all the data assessed in the study were obtained as part of routine clinical assessments from patients' medical records, written consent was not obtained, in accordance with the guidelines of the Chinese Ministry of Health.

\section{Data Collection}

Four experienced palliative care attending physicians who received training participated in the retrospective collection of patients' medical information through electronic chart review. Electronic medical chart is recorded in the Union Medical System (UMS) of FUSCC, which is an electronic medical record system that contains all medical information of patients in FUSCC, including demographic and clinical data. The following variables were extracted into SPSS software: (1) general baseline demographic characteristics-including age, sex, indigenous (Shanghainese) or not, medical insurance status, and area of residence (rural or urban according to the address of patients' permanent residence); (2) disease-related characteristics-including cancer diagnosis, pathology, gene detection report, metastatic sites and previous treatment.

Pain and symptom assessment 
$\mathrm{CP}$ was defined as pain directly because of the tumor presence or secondary to anti-enoplastic treatment. The Brief Pain Inventory (BPI) was used to evaluate CP[10].. The BPI scale is an assessment tool for cancer related pain which has been translated into Chinese. Pain intensity was measured with the Numeric Scoring Scale (NRS), which ranged from 0 to $10(0=$ no pain, $10=$ worst possible pain). For analysis, pain intensity was categorized into no pain (NRS 0) mild pain (NRS 1-3) and moderate(NRS 46)to severe pain (NRS 7-10). Pain medication included both classic analgesics, such as opioids, paracetamol and NSAIDs; and co-analgesics, such as steroids, tri-cyclic antidepressants and antiepileptics. The BPI survey was assessed at the admission of patients.

\section{Statistical Analysis}

Patients characteristics and disease features were analyzed by descriptive statistics such as proportions,means,standard deviations, medians, and mode. $95 \%$ confidence intervals $(95 \% \mathrm{Cl})$ were calculated for median length. SPSS software (v.16.0) was employed for statistical analysis. $p$ value less than 0.05 was considered to be significant.

\section{Results:}

\section{general and disease related characteristics}

The median age was 66 years(range $25-85,95 \% \mathrm{Cl}$ : 61.68-66.76) with mean age of 64.22 years. $68.4 \%$ $(n=60)$ were male, 63.2\% $(n=60)$ were Shanghaiese (indigenous). $66.3 \%(n=63)$ patients were covered with local National Health Medical insurance. Eastern Cooperative Oncology Group (ECOG) performance status was > $=3$ among $75.7 \%(n=72)$ patients. Adenocarcinoma (ADC) was the most popular pathology among NSCLC $(n=56,58.9 \%)$, with $(n=13,13.7 \%)$ squamous cell carcinoma (SqCC). Among 56 patients with gene expression, 27 patients were testified EGFR mutation positive and 3 patients were testified ALK mutation positive. Most of the male sample (51/65, 78.4\%) had a history of smoking. Most of the patients stopped smoking after diagnosis $(48 / 53,90.6 \%)$. Only 5 patients reported smoking currently. The most prevalent visceral metastatic site was bone $(n=57,60 \%)$, more than two sites were detected as visceral metastasis among $62.1 \%(n=59)$ patients. $53.7 \%$ patients $(n=51)$ reported plural effusion. Before referral to $\mathrm{PC}, 12$ patients received surgery (12.6\%), 43 patients received chemotherapy $(45.3 \%)$. $62.1 \%(n=59)$ patients received no anti-cancer treatments since their disease diagnoses. Details were shown in Table 1. 
Table 1

gengeral and disease related characteristic of 95 advanced lung cancer patients at the enrollment of PCU

\begin{tabular}{|c|c|c|c|c|c|}
\hline Variables & $\mathbf{N}$ & $\%$ & Variables & $\mathbf{N}$ & $\%$ \\
\hline Age & & & Pathology & & \\
\hline$>65$ & 48 & 50.5 & NSCLC & 78 & \\
\hline$<=65$ & 47 & 49.5 & $A D C$ & 56 & 58.9 \\
\hline Gender & & & $\mathrm{SqCC}$ & 13 & 13.7 \\
\hline Male & 65 & 68.4 & Others NSCLC & 9 & 9.5 \\
\hline Female & 30 & 31.6 & SCLC & 2 & 2.1 \\
\hline Living location & & & Without pathology & 15 & 15.8 \\
\hline Shanghai & 60 & 63.2 & Stage & & \\
\hline Non-shanghai & 35 & 36.8 & III & 3 & 3.2 \\
\hline ECOG & & & IV & 80 & 84.2 \\
\hline$<=2$ & 23 & 24.3 & cannot be staged & 10 & 10.5 \\
\hline$>=3$ & 72 & 75.7 & extensive(SCLC) & 2 & 2.1 \\
\hline Medical insurance & & & Gene expression & & \\
\hline yes & 68 & 71.8 & Yes & 56 & 58.9 \\
\hline no & 27 & 28.4 & EGFR & 27 & 48.2 \\
\hline Living area & & & ALK & 3 & 5.35 \\
\hline Urban & 67 & 70.5 & Not test & 39 & 60.0 \\
\hline Rural & 28 & 29.5 & Combination & & \\
\hline Smoking & & & Atelectasis & 21 & 22.1 \\
\hline Never & 44 & 46.3 & emphysema & 24 & 25.3 \\
\hline Ex-smoker & 48 & 50.5 & pneumonia & 28 & 29.5 \\
\hline Current & 5 & 5.26 & pre-referral treatmentl & & \\
\hline unknown & 3 & 3.16 & Any anti-cancer therapy & 59 & 62.1 \\
\hline Metastasitc & & & Surgeon & 12 & 12.6 \\
\hline Lymphon metastastic & 68 & 28.4 & chemotherapy & 43 & 45.3 \\
\hline
\end{tabular}

Abbreviations: ADC, adenocarcinoma; SqCC, squamous cell carcinoma; NSCLC, non-small cell carcinoma; \#Others: others include indwelling pleural catheters for malignant pleural effusion, transcatheter arterial chemoembolization (TACE) etc. 


\begin{tabular}{|llllll|}
\hline Variables & $\mathbf{N}$ & $\mathbf{\%}$ & Variables & $\mathbf{N}$ & $\%$ \\
\hline liver & 20 & 21.1 & Radiotherapy & & \\
\hline Bone & 57 & 60.0 & chest & 13 & 13.7 \\
\hline brain & 23 & 24.2 & brain & 6 & 6.3 \\
\hline Pleural effussion & 51 & 53.7 & bone & 27 & 28.4 \\
\hline$>=$ two visceral & 59 & 62.1 & EGFR-TKI & 14 & 14.7 \\
\hline & & & Others\# & 36 & 37.9 \\
\hline & & & No anti-cancer therapy & \\
\hline $\begin{array}{l}\text { Abbreviations: ADC, adenocarcinoma; SqCC, squamous cell carcinoma; NSCLC, non-small cell } \\
\text { carcinoma; \#Others: others include indwelling pleural catheters for malignant pleural effusion, } \\
\text { transcatheter arterial chemoembolization (TACE) etc. }\end{array}$ \\
\hline
\end{tabular}

\section{Pain characteristics and related factors}

Of all 95 included patients, 54 patients (56.7\%) reported cancer pain suffering, the intensity of pain ranged from 0 to 9 . Of 54 patients with pain, there were 23 patients $(42.6 \%)$ who suffered from severe pain (score > = 7) at the admission of PC. The mean pain intensity (NRS) for 54 patients was 5.2 ( $95 \mathrm{Cl} \%$ : 4.62-5.79). The median NRS score for these patients was 6 . These 54 patients were analyzed in more details. Chest pain was reported by $40.7 \%(n=22)$ of patients with the mean 5.27 ; pain in dorsum was reported by $20.4 \%(n=11)$ with the mean socre 5.36 . Of these patients who reporting pain, 34 patients $(63 \%)$ used drugs which were included in the NCCN guideline for cancer pain. Despite $56.7 \%$ of the patients referred some pain at baseline, only $61.1 \%(n=33)$ patients assumed analgesics including weak and strong opioids. Details were shown in Table 2. 
Table 2

Initial pain characteristics of 54 patients suffered pain

\begin{tabular}{|c|c|c|c|c|c|}
\hline Variable & Number & $\%$ & Variable & $\mathbf{N}$ & $\%$ \\
\hline Pain (yes/no) & \multicolumn{2}{|l|}{$5.2($ mean) } & \multicolumn{3}{|l|}{ Pain medication * } \\
\hline No pain & 41 & 43.2 & Yes & 34 & 63.0 \\
\hline Mild $<=3$ & 14 & 14.7 & No & 20 & 37 \\
\hline Moderate(4-6) & 17 & 17.9 & \multicolumn{3}{|l|}{ Body location \# } \\
\hline Severe $(7-10)$ & 23 & 24.2 & Head, Neck,shoulder & 4 & 7.4 \\
\hline \multicolumn{3}{|l|}{ Pre-referral med } & Chest & 22 & 40.7 \\
\hline NSAIDs & 1 & 1.9 & Back & 11 & 20.4 \\
\hline Weak opioids & 15 & 27.8 & Abdomen (include stomach) & 4 & 7.4 \\
\hline Strong opioids & 18 & 33.3 & Arms,hands $\square$ feet & 8 & 14.8 \\
\hline TCA\&AE & 2 & 3.8 & Others \& & 5 & 9.3 \\
\hline \multicolumn{6}{|c|}{ Pain medication * : paients received pain medication at the admission of PC referral } \\
\hline \multicolumn{6}{|c|}{$\begin{array}{l}\text { * WHO's cancer pain ladder for adults: NSAIDs include aspirin and paracetamol, coxibs. Weak opioids } \\
\text { include codeine, dextropropoxyphene, and tramadol. Strong opioids include morphine, oxycodone, } \\
\text { fentanyl, buprenorphine and ketobemidone. TCA, tricycle antidepressants, AE, antiepileptics \# The } \\
\text { percentage was not calculated because lots of patients had more than two pain sites. }\end{array}$} \\
\hline \multicolumn{6}{|c|}{ \& Others: include pelvis, hip and whole body pain } \\
\hline
\end{tabular}

\section{Relationship between pain characteristics and main baseline characteristics}

There was a significant difference in the frequency of pain between patients with and without bone metastasis $(p=0.006)$, between patients with more than two sites and non-more than two sites metastasis $(p=0.032)$. Patients received EGFR-Tyrosine kinase inhibitors (EGFR-TKI) had lower pain prevalence $(p=0.033)$. For pain intensity, Patients with bone metastases $(p=0.005)$ and those with more than two metastases $(p=0.022)$ had higher mean scores of pain, with significant differences. For CRP index with 11 as the boundary, there was a significant difference in the mean score of pain intensity $(p=$ 0.012). Although there was no statistical difference in the results, patients who had used EGFR-TKI had a lower mean pain score. Details were shown in Table 3. 
Table 3

Relationship between pain and main baseline characteristics

\begin{tabular}{|c|c|c|c|c|c|c|c|}
\hline Variables & Pain & No pain & p value & Variables & Pain & No pain & $\mathrm{p}$ value \\
\hline Age & & & 0.409 & Metastasis & & & \\
\hline$>=65$ & 25 & 23 & & Lymphon & 44 & 32 & 0.797 \\
\hline$<65$ & 29 & 18 & & Liver & 14 & 6 & 0.212 \\
\hline Gender & & & 0.504 & Bone & 39 & 18 & $0.006^{*}$ \\
\hline Male & 35 & 30 & & Brain & 12 & 11 & 0.635 \\
\hline Female & 19 & 11 & & $>=$ two visceral & 39 & 20 & $0.032 *$ \\
\hline Smoking & & & 0.409 & Pleural effussion & 27 & 24 & 0.533 \\
\hline Yes & 25 & 23 & & $\operatorname{WBC}(10 \times 9 / \mathrm{L})$ & & & 0.516 \\
\hline No & 29 & 18 & & $>10$ & 17 & 12 & \\
\hline EGFR & & & 0.248 & $<=10$ & 37 & 29 & \\
\hline mutation & 15 & 12 & & $\mathrm{Neu}(10 \times 9 / \mathrm{L})$ & & & \\
\hline no mutation & 19 & 10 & & CRP & & & 0.553 \\
\hline Pre-referral treat & & & & $>=11$ & 39 & 27 & \\
\hline Surgeon & 6 & 6 & 0.757 & $<11$ & 15 & 14 & \\
\hline Chemotherapy & 28 & 15 & 0.152 & & & & \\
\hline Radiotherapy & 19 & 10 & 0.369 & & & & \\
\hline EGFR-TKI & 7 & 20 & $0.033^{*}$ & & & & \\
\hline${ }^{*} p<0.05$ & & & & & & & \\
\hline
\end{tabular}


Table 4

Pain intensity difference with variables

\begin{tabular}{|lll|}
\hline Variables & mean (SD) & p value \\
\hline Bone metastasis & & $0.005^{*}$ \\
\hline Yes & $5.74(1.624)$ & \\
\hline No & $4.07(1.996)$ & $0.022^{*}$ \\
\hline$>=$ two visceral & & \\
\hline Yes & $5.67(1.710)$ & \\
\hline No & $4.27(2.030)$ & \\
\hline CRP & & \\
\hline$>11$ & $5.63(1.777)$ & $0.012^{*}$ \\
\hline$<=11$ & $4.62(2.567)$ & \\
\hline EGFR-TKI\& & $2.81(3.087)$ & \\
Yes & $3.56(2.847)$ & \\
\hline No & \\
\hline * t test $p<0.05$ & \\
\hline \& EGFR-TKI includes currently available gefitinib, erlotinib, ositinib, etc \\
\hline
\end{tabular}

\section{Discussion:}

Our study showed the general characteristics, pain and related factors of patients who referraled to Palliative care service of advanced NSCLC patients. It is a relatively rare description on the characteristics of pain caused by a certain cancer. In addition to reflecting patient's pain features, we try to find some indicators and factors that can reflect patient's pain frequency and its' severity in clinical data such as inflammatory markers, related symptom severity. Our study confirmed that the clinical manifestations of pain in NSCLC patients were correlated with bone metastasis, metastasis with more than two sites, elevated CRP, and the EGFR-TKI usage. Whether there is a certain potential connection between these factors deserves to be further studied.

Of all 95 included patients, 54 patients (56.7\%) reported cancer pain suffering. Of 54 patients with pain, there were 23 patients $(42.6 \%$ ) who suffered from severe pain (score $>=7$ ) at the admission of PC. The incidence of pain in lung cancer patients was reported quite differently. It may be due to different baseline conditions of patients, different research background, etc. Prevalence of pain in the early stages of lung cancer has been reported to be as high as $56 \%$ [11]. Over $90 \%$ of patients with advanced lung cancer experienced pain and fatigue in another research [12]. In another study of patients with NSCLC, pain was 
reported to be only $37 \%$ [13]. These studies were quite heterogeneous with differences in sample sizes, cancer stage and clinical variables, making it difficult to ascertain the exact prevalence of pain among advanced lung cancer patients.

In our patients' baseline, at the time of admission to $\mathrm{PC}, 60 \%$ of patients had been confirmed combined with bone metastasis. Approximately $40 \%$ of NSCLC patients developed bone metastases in their disease course [14]. Previous studies have testified that bone metastases strongly affected presence of cancer pain[15]. Bone pain was thought to be related with stimulation of sensory fibers distributed in the periosteum. Osteodynia was also known as related with prostaglandins, endothelins, nerve growth factors or inflammatory cells, and maybe the activation of several acid sensing receptors by local tissue acidosis[16,17]. The presence of bone metastasis also affects the pain distribution of NSCLC patients. Bone metastases sites of lung cancer are most common in the lumbar spine, pelvis, thoracic spine and ribs. In our research, the most frequent pain location was chest $(n=22)$, followed by dorsum $(n=11)$. The incidence of dorsum is high in patients, which may be related to the proportion of bone metastasis of our patients.

With the development of targeted therapy, more patients have done the corresponding gene detection before receiving systematic treatment. There was no significant correlation between EGFR mutation status and pain characteristics in our research but patients used EGFR-TKI reported lower pain intensity with statistical differences. Previous research also showed that EGFR-TKI strongly reduced nocifensive behavior in mouse models of inflammatory and chronic pain. The PI3K/AKT/mTOR pathway and upregulated mitogen-activated protein kinase (MAPK) expression maybe involved in enhancement of nociception by activation of EGFR [18]. No EGFR-TKI usage, together with smoking, histological subtype and performance status were shown as associated with bone metastasis' skeletal-related events (SRS) [19]. The systemic effect and mechanism of EGFR mutation and EGFR-TKI usage, their relationship with bone metastasis and pain characteristics are worth of further study.

$C$ reactive protein (CRP), together with inflammation markers were testified to be related with pain manifestaion in previous research $[20,21]$. Among breast cancer patients, women with pain detected higher CRP levels before anticancer treatment than women without pain[22]. It was reported that systemic inflammation maybe linked $\mathrm{CP}$, but the relationship between pain and systemic inflammation in cancer was less well understood $[23,24$

]. The current findings suggest a potential association between pain and inflammatory processes in NSCLC patients, with potential attention for future research and treatment strategies.

\section{Limitation}

Due to the limited number of cases, the specific EGFR mutation sites of patients, the diversity of different generation of EGFR-TKI drugs used, and other gene mutations of NSCLC patients were not included in analysis. The further relationship between specific drugs, cycles, and pain performance in patients using EGFR-TKI will be worth further study. 


\section{Conclusion:}

Our study showed the general characteristics, pain performance and related factors of advanced NSCLC patients who referral to palliative care service. This study confirmed that these patents' clinical manifestations of pain were correlated with bone metastasis, metastasis with more than two sites, elevated CRP, and the EGFR-TKI usage. Whether there is a certain potential connection between these factors deserves further study.

\section{Declarations}

\section{Authors' contributions}

Xiaoli Gu, Huifeng Gao, Menglei Chen, Minghui Liu, Zhe Zhang and Wenwu Cheng participated in the conception and design of the study; Xiaoli Gu and Wenwu Cheng monitored data collection; Xiaoli Gu and Huifeng Gao analyzed data; Menglei Chen, Minghui Liu, Zhe Zhangparticipated in data interpretation, drafting, and revising the manuscript. All authors read and approved the final manuscript.

\section{Conflict of interest}

The authors declare that they have no conflict of interest.

\section{Acknowledgments}

We acknowledge and send our deepest thanks to the patients and caregivers who participated in this study.

\section{Funding information:}

This research was supported by Shanghai Pain Research foundation for Young, Shanghai Medical Association.

\section{Ethics approval and consent to participate}

Approval to perform the study has been obtained from the ethics committee of the Fudan University Shanghai Cancer Center. Participation was voluntary and anonymous. Informed consent was obtained written at the beginning of admission.

\section{Referrence}

1. Dai W , Zhang $Y$, Feng $W$, Liao X, Mu Y , Zhang R, et al. Using patient-reported outcomes to manage postoperative symptoms in patients with lung cancer: Protocol for a Multicentre, Randomised Controlled Trial. BMJ Open.2019;9: e030041.

2. Walling AM , Weeks JC, Kahn KL, Tisnado DKeating NL, Dy SM , et al. Symptom Prevalence in Lung and Colorectal Cancer Patients. J Pain Symptom Manage. 2015;49: 192-202. 
3. McGee SF , Zhang T , Jonker H, Laurie SA , Goss G, Nicholas G et al. The Impact of Baseline Edmonton Symptom Assessment Scale Scores on Treatment and Survival in Patients With Advanced Non-small-cell Lung Cancer.Clin Lung Cancer. 2018;19: e91-e99.

4. Marie Fallon, Jane Walker,Lesley Colvin,Aryelly Rodriguez, Gordon Murray, Michael Sharpe, et al. Pain Management in Cancer Center Inpatients: A Cluster Randomized Trial to Evaluate a Systematic Integrated Approach-The Edinburgh Pain Assessment and Management Tool. J Clin Oncol. 2018;36:1284-1290.

5. RE Coleman. Skeletal Complications of Malignancy. Cancer.1997; 80: 1588-94.

6. NCCN Guidelines Insights: Non-Small Cell Lung Cancer, Version 1.2020.

7. Kersten C , Cameron MG. Cetuximab Alleviates Neuropathic Pain Despite Tumour Progression. BMJ Case Rep.2012;14.

8. Kersten C , Cameron MG , Laird B , Mjåland S . Epidermal Growth Factor Receptor-Inhibition (EGFR-I) in the Treatment of Neuropathic Pain. Br J Anaesth. 2015; 115 :761-7.

9. Van den Beuken-van Everdingen MH, De Rijke JM, Kessels AG,HC Schouten, M van Kleef, J Patijn. Prevalence of Pain in Patients With Cancer: A Systematic Review of the Past 40 Years. Ann Oncol.2007; 18: 1437-49.

10. CS Cleeland, K M Ryan. Pain Assessment: Global Use of the Brief Pain Inventory.Ann Acad Med Singapore.1994; 23:129-1 of non-small cell lung cancer in the USA: a real-world cross-sectional study. Support Care Cancer.2014;22: 181-7.

13. McGee SF, Zhang T, Jonker H, Laurie SA, Goss G, Nicholas $G$ et al. The Impact of Baseline Edmonton Symptom Assessment Scale Scores on Treatment and Survival in Patients With Advanced Nonesmall-cell Lung Cancer. Clin Lung Cancer. 2018;19; e91-e99.

14. Oliveira MB , Mello FC, Paschoal ME. The relationship between lung cancer histology and the clinicopathological characteristics of bone metastases. Lung Cancer.2016;96:19-24.

15. Saito M , Kage H, Ando T, Sawada R, Amano Y , Goto Y, et al. Prevalence of Bone Pain Decreases as Lymph Node Stage Increases in Nonsmall Cell Lung Cancer Patients. Curr Probl Cancer. 2019;43: 86-91.

16. Falk S , Dickenson AH. Pain and Nociception: Mechanisms of Cancer-Induced Bone Pain. J Clin Oncol.2014; 32: 1647-54.

16. Ghilardi JR, Röhrich H, Lindsay TH, Sevcik MA, Schwei MJ, Kubota K et al .Selective Blockade of the Capsaicin Receptor TRPV1 Attenuates Bone Cancer Pain J Neurosci.2005; 25: 3126-31.

17. 18. Martin LJ , Smith SB , Khoutorsky A, Magnussen CA , Samoshkin A, Sorge RE , et al. Epiregulin and EGFR Interactions Are Involved in Pain Processing. J Clin Invest.2017;127:3353-3366.

18. Sun JM , Ahn JS, Lee S, Kim JA, Lee J, Park YH, et al. Predictors of Skeletal-Related Events in NonSmall Cell Lung Cancer Patients With Bone Metastases. Lung Cancer actions. 2011;71:89-93.

19. Kwekkeboom KL, Tostrud L, Costanzo E, Coe CL, Serlin RC, Ward SE et al. The Role of Inflammation in the Pain, Fatigue, and Sleep Disturbance Symptom Cluster in Advanced Cancer. J 
Pain Symptom Manage. 2018; 55:1286-1295.

20. Laird BJ , Scott AC, Colvin LA, McKeon AL, Murray GD, Fearon KC, et al. Cancer Pain and Its Relationship to Systemic Inflammation: An Exploratory Study. Pain. 2011;152: 460-463.

21. Starkweather AR, Lyon DE, Schubert CM. Pain and Inflammation in Women With Early-Stage Breast Cancer Prior to Induction of Chemotherapy. Biol Res Nurs.2013;15:234-241.

22. Oliveira KG , von Zeidler SV , Lamas AZ, Podestá JR, Sena A, Souza ED ,et al. Relationship of Inflammatory Markers and Pain in Patients With Head and Neck Cancer Prior to Anticancer Therapy. Braz J Med Biol Res. 2014;47: 600-604.

23. Laird BJ , Scott AC, Colvin LA, McKeon AL, Murray GD, Fearon KC, et al. Cancer Pain and Its Relationship to Systemic Inflammation: An Exploratory Study. Pain.2011;152:460-3. 